\title{
Electronic and electrical properties of functional interfaces studied by hard X-ray photoemission
}

\author{
A. Zenkevich ${ }^{\mathrm{a}, \mathrm{b}, *}$, Y. Matveyev $^{\mathrm{b}}$, M. Minnekaev $^{\mathrm{b}}$, Yu. Lebedinskii $^{\mathrm{b}}, \mathrm{S}$. Thiess ${ }^{\mathrm{c}}, \mathrm{W}$. Drube ${ }^{\mathrm{c}}$ \\ a National Research Center "Kurchatov Institute", NBICS-Center, 123182 Moscow, Russia \\ b NRNU "Moscow Engineering Physics Institute", 115409 Moscow, Russia

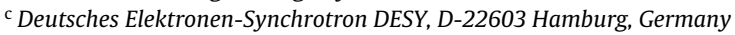

\section{A R T I C L E I N F O}

\section{Article history:}

Available online $\mathrm{xxx}$

\section{Keywords:}

Hard X-ray photoelectron spectroscopy

Electronic band line-up

Electrical properties

Functional multilayered structures

Non-volatile memory devices

\begin{abstract}
A B S T R A C T
As the device downscaling in nanoelectronics has reached the $10 \mathrm{~nm}$ range, the functionality of materials employed in multilayered structures to be used in future logic and memory devices is largely defined by their interface properties. In particular, the electrical properties of the functional stacks are directly related to the electronic band line-up which is affected by the electric dipoles building up at the interface(s). In this work, hard X-ray photoelectron spectroscopy is applied to probe the electronic conditions at the interfaces of several relevant multilayered functional structures and to correlate the results with their electrical (transport) properties.
\end{abstract}

(c) 2013 Elsevier B.V. All rights reserved.

\section{Introduction}

The study of complex heterostructures comprising oxide layers has attracted much attention because of their intriguing properties which are not only fundamentally interesting but also often very promising for potential device applications [1]. As the thickness of oxide materials employed in such multilayered structures drops beyond the $10 \mathrm{~nm}$ scale, new functionalities emerge giving rise to novel concepts of logic and memory devices. In particular, a range of phenomena referred to as "resistive switching" effects in metal oxide based metal-insulator-metal (MIM) multilayers has been identified and is currently being investigated targeting the development of the novel non-volatile memory applications. Hard X-ray photoelectron spectroscopy (HAXPES) combining brilliant $\mathrm{X}$-ray undulator radiation from 3rd generation sources with commercially available optimized high-energy electron spectrometers has recently emerged as an excellent tool providing information on the chemical and electronic structure of prototype functional oxide based multilayered stacks thereby enabling new insight into the physical mechanisms behind the resistance switching effects in ultrathin metal oxide films [2-4]. The use of hard X-rays in an energy range of about $2.5-12 \mathrm{keV}$ is particularly beneficial since it produces photoelectrons with variable high kinetic energies yielding large electron inelastic mean free paths which results in electron escape depths up to about $20 \mathrm{~nm}$. This technique is

\footnotetext{
* Corresponding author at: National Research Center "Kurchatov Institute", NBICS-Center, 123182 Moscow, Russia. Tel.: +7 9036874945.

E-mail address: avzenkevich@mephi.ru (A. Zenkevich).
}

therefore ideally suited to non-destructively probe both the chemical composition and the electronic structure of multilayered structures as well as buried interfaces at some $10 \mathrm{~nm}$ depths [5]. Functional structures often comprise top metal layers (serving as electrodes), and it is worth noting that continuous metal layers can only reliably be produced with thicknesses larger than 7-10 nm. It is therefore not possible to use soft X-ray electron spectroscopy methods or laboratory XPS instruments for such studies. In addition, HAXPES provides a unique opportunity to monitor the spatial redistribution of charges across the functional structures upon ex situ or in situ voltage biasing conditions, modeling the devices under operation. In this work, we report on the application of HAXPES instruments at DORIS III and PETRA III (DESY, Hamburg) to investigate the electronic and electrical properties at the interfaces of several relevant functional structures having potential for applications in nanoelectronics and spintronics.

The materials under investigation here are prototypical functional multilayers representing alternative emerging non-volatile memory concepts. In particular, a promising concept challenging currently dominating Flash technology exploits reversible switching under external voltage between the conducting and insulating states in ultrathin films of various materials embedded in a metalinsulator-metal (MIM) structure (see review [6] and refs. therein). The challenge is to elucidate the microscopic physical mechanism(s) behind the observed switching phenomena which will enable to optimize the operation of future memory devices based on resistive switching which have entered the commercialization stage. Among the materials exhibiting resistive switching behavior are transition metal oxides, particularly hafnium oxide. However, devices constructed using $\mathrm{HfO}_{2}$ often suffer from nonuniformity 
of resistive switching parameters such as the on-/off-state resistance values and $V_{\text {set }} / V_{\text {reset }}$ switching voltages; this nonuniformity leads to false programming and gives rise to readout hazards. Earlier, it has been shown that the introduction of an additional $\mathrm{ZrO}_{x}$ layer leads to a tight distribution of switching parameters, good switching endurance, and good data retention [7]. Furthermore, the bilayer $\mathrm{HfO}_{2-x} / \mathrm{ZrO}_{x}$ structure shows lower reset current and operating voltage than $\mathrm{HfO}_{x}$ single layer under dc sweep voltage. In this paper, HAXPES is used to probe functional MIM structures based on $\mathrm{p}^{++}-\mathrm{Si} / \mathrm{HfO}_{2-x} / \mathrm{ZrO}_{x} / \mathrm{Pt}$ upon in situ biasing ("under operation"). By observing small changes of the core level lines position following the assumed (charged) oxygen vacancies drift we get insight into the processes accompanying the effect of resistive switching in dielectrics based on the transition metal oxides. HAXPES technique has been previously employed to investigate the origin of the resistivity change in thin films, particularly, in $\mathrm{NiO}$ [8].

In an alternative memory concept employing ultrathin ferroelectric (FE) layers in metal/FE/metal heterostructures, the predicted effect of ferroelectric polarization orientation on the electron transport properties in the so-called ferroelectric tunnel junctions (FTJ) [9] has recently been directly established [10,11]. The results clearly indicate that the tunneling electroresistance which depends on the electrostatic potential barriers at both $\mathrm{FE} /$ metal electrode interfaces is controlled by the direction of the FE polarization. One of the promising though rather unexplored material systems is $\mathrm{Pt} / \mathrm{BaTiO}_{3}$ because due to the favorable lattice mismatch $\left[\left(a_{\mathrm{BTO}}-a_{\mathrm{Pt}}\right) / a_{\mathrm{Pt}} \approx 1.8 \%\right]$ an ultrathin heteroepitaxial $\mathrm{BaTiO}_{3}$ layer on $\mathrm{Pt}$ is under biaxial compressive strain, which produces tetragonal distortion and ensures that the ferroelectric polarization is aligned in the direction perpendicular to the surface [12]. In this work, HAXPES is employed to directly determine the electronic band line-up in the functional FTJ based on the $\mathrm{Pt} / \mathrm{BaTiO}_{3} / \mathrm{Cr}$ heterostructure and eventually to reconstruct the electrostatic potential barrier profile to correlate their electronic and transport properties. We also investigate the expected effect of oxygen vacancies in $\mathrm{BaTiO}_{3}$ on the band alignment at the $\mathrm{Pt} / \mathrm{BaTiO}_{3}$ interface.

Further extension of an FTJ concept is to use ferromagnetic electrodes to exploit the so-called "interfacial magnetoelectricity", i.e. magnetoelectric coupling arising at a ferromagnetic metal/dielectric (ferroelectric) interface [13]. Such heterointerfaces have proven to be ideal for controlling and manipulating electrical charges and spins in multifunctional solid state devices, and recently the successful implementation of ferroelectric control of spin polarization has been convincingly demonstrated [14]. To elucidate the effect of FE polarization on the magnetic and transport properties, it is important to obtain detailed information on the interfacial electronic structure. Here, using HAXPES we experimentally determine the electronic band alignment at the $\mathrm{La}_{0.67} \mathrm{Sr}_{0.33} \mathrm{MnO}_{3}$ (LSMO)/BaTiO 3 interface which is a model ferromagnetic/FE system.

\section{Experimental}

\subsection{HAXPES instruments}

The HAXPES data presented here were measured using two different instruments at DESY (Hamburg, Germany) synchrotron photon sources. At the DORIS III storage ring, the X-ray photoemission setup at wiggler beamline BW2 was used which provides a high-flux X-ray beam in an energy range from 2.4 to $8 \mathrm{keV}$. For this experiment, an X-ray energy of $4.5 \mathrm{keV}$ was chosen at this beamline. Photoelectrons were recorded using a SCIENTA SES-200 analyzer. This facility allows to obtain high quality data with medium energy resolution around $0.5 \mathrm{eV}$ with a $\mathrm{Si}(111)$ double-crystal monochromator which is sufficient for most core level studies. The size of the focused X-ray beam at the sample is rather large $\left(\sim 0.4 \times 2 \mathrm{~mm}^{2}\right)$.

The second instrument used in this study is the HAXPES endstation at X-ray undulator beamline P09 of the new 3rd generation $\mathrm{X}$-ray storage ring PETRA III [15]. Typical spot sizes on the sample are $0.1 \mathrm{~mm} \times 0.3 \mathrm{~mm}$. Here, the spectrometer is a SPECS Phoibos 225 allowing to acquire electron spectra up to $15 \mathrm{keV}$ kinetic energy. The data shown here were measured using the Si(311) highheatload double-crystal monochromator tuned to $6 \mathrm{keV}$ resulting in an overall energy resolution of about $0.2 \mathrm{eV}$.

At either instrument, the spectrometer energy scale was calibrated using the $\mathrm{Au} 4 f$ line at $\mathrm{BE}_{\mathrm{Au} 4 f}=84.0 \mathrm{eV}$. The UNIFIT software was used to fit the experimental data [16].

For the 5-axis precision manipulator of the P09 HAXPES system, a specialized ultra-high vacuum (UHV) compatible sample holder with 4 electrical contacts has been designed to allow in situ voltage biasing of the samples, enabling also to acquire spectra from the biased samples. This option provides an opportunity to monitor the changes in the chemical and electronic structure of the functional structures modeling devices under operation. The constant voltage as well as the bias sweeps to the samples were applied using an Agilent B2912A precision source/measure unit.

\subsection{HAXPES methodology of band line-up determination at the metal/FE interface}

The value of the electric potential barrier height $\varphi$ (also known as a conduction band offset, $\mathrm{CBO}$ ) at the metal/FE interface(s) is an important characteristics, particularly to predict the transport properties of the respective ferroelectric tunnel junction. It is defined as $\varphi=E_{\mathrm{g}}-\mathrm{VBO}_{\mathrm{Me} / \mathrm{BTO}}$ where $E_{\mathrm{g}}$ is the band gap of the FE and $\mathrm{VBO}_{\mathrm{Me} / \mathrm{BTO}}$ is the valence band offset, i.e. the energy separation between the FE valence band maximum (VBM) and the Fermi level of the metal in contact. In order to determine the band line-up at the metal/BaTiO 3 interface we employ the well-known methodology described previously $[17,18]$. In particular, the VBO at the $\mathrm{Pt} / \mathrm{BaTiO}_{3}$ interface is calculated according to the formula (1):

$$
\begin{aligned}
& \mathrm{VBO}=\left(E_{\mathrm{Ti} 2 p 3 / 2}-E_{\mathrm{Pt} 4 f 7 / 2}\right)_{\mathrm{BTO} / \mathrm{Pt}}+\left(E_{\mathrm{Pt} 4 f 7 / 2}-E_{\mathrm{F}}\right)_{\mathrm{Pt}} \\
& -\left(E_{\mathrm{Ti} 2 p 3 / 2}-\mathrm{VBM}\right) \mathrm{BTO}
\end{aligned}
$$

where the quantities $\left(E_{\mathrm{Pt} 4 f 7 / 2}-E_{\mathrm{F}}\right),\left(E_{\mathrm{Ti} 2 p 3 / 2}-\mathrm{VBM}\right)_{\mathrm{BTO}}$ and $\left(E_{\mathrm{Pt} 4 f 7 / 2}-E_{\mathrm{Ti} 2 p 3 / 2}\right)$ are measured by HAXPES on clean bulk Pt, $\mathrm{BaTiO}_{3}$ and $\mathrm{Pt} / \mathrm{BaTiO}_{3}$ bi-layers, respectively. Similar measurements can be performed for the $\mathrm{Fe} / \mathrm{BaTiO}{ }_{3}$ bi-layers. Once the band gap $E_{\mathrm{g}}$ of $\mathrm{BaTiO}_{3}$ films is determined independently, the value of the potential barrier height $\varphi$ is obtained.

\subsection{Sample preparation}

$\mathrm{p}^{++}-\mathrm{Si} / \mathrm{HfO}_{2-x} / \mathrm{ZrO}_{x} / \mathrm{Pt}$ multilayered stacks were grown by the combination of Atomic Layer Deposition (ALD) and Pulsed Laser Deposition (PLD) techniques. Details of the deposition procedure are described elsewhere [19]. Briefly, $\mathrm{HfO}_{2}$ layers were grown by ALD on highly doped $(\rho=0.01 \mathrm{Ohm} \mathrm{cm}) \mathrm{p}^{++}$-Si substrates $\left(T_{\text {subst. }}=300^{\circ} \mathrm{C}\right)$, while ultrathin $\mathrm{Zr}$ and Pt layers were separately deposited on top by PLD in a single vacuum cycle at room temperature. The thickness of the $\mathrm{Zr}$ interlayer $d \approx 4 \mathrm{~nm}$ was optimized in terms of the resistive switching effect by separately performing $I-V$ measurements on standard size $\left(\sim 10^{-4} \mathrm{~mm}^{2}\right)$ MIM stacks (results will be reported elsewhere). The thickness of the Pt capping layer serving as the top electrode also has to be carefully chosen to ensure both a continuous film coverage and a sufficiently high yield of photoelectrons from the buried $\mathrm{HfO}_{2-x} / \mathrm{ZrO}_{x} / \mathrm{Pt}$ 
interfaces. It has been previously shown that in a PLD process a continuous layer of noble metals on dielectric substrates can be achieved at coverages as small as $6 \mathrm{~nm}$ [20], and hence the thickness 7-10 nm was adopted for the Pt overlayer in our experiments. However, an ultrathin Pt electrode has a surface conductivity comparable with that across the $\mathrm{HfO}_{2}$ functional layer. The substantial electric potential distribution across the sample area upon biasing can result in fluctuations of the resistive switching phenomena in the $\mathrm{HfO}_{2}$ functional layer. To account for this detrimental effect, the potential distribution across the $\mathrm{Pt} / \mathrm{HfO}_{2} / \mathrm{Si}$ stack was modeled using the electric resistivity values for both metal and dielectric layers measured for our experimental conditions. It was found that for the adopted experimental conditions the allowable maximal potential drop $\Delta U=0.1 \mathrm{~V}$ at $U=5 \mathrm{~V}$ across the $\mathrm{p}^{++}-\mathrm{Si} / \mathrm{HfO}_{2} / \mathrm{ZrO} \mathrm{P}_{x} / \mathrm{Pt}$ sample area is achieved at a lateral distance $d \approx 0.1 \mathrm{~mm}$. Therefore, to obtain a uniform potential distribution across the sample area of $2 \mathrm{~mm} \times 2 \mathrm{~mm}$ during biasing, a mesh of thick $(\sim 100 \mathrm{~nm})$ $0.1 \mathrm{~mm}$ wide conductive metallic ( $\mathrm{Al}$ or $\mathrm{Cu}$ ) stripes with a spacing of $\sim 0.1 \mathrm{~mm}$ were deposited through a shadow mask on top of the ultrathin Pt electrode as illustrated in Fig. 1.

The employed geometry provides the opportunity to monitor the subtle changes in the chemical and electronic structure both in the bulk of individual layers and at the interfaces of the multilayer structure following in situ biasing of the sample. Obviously, by using such a sample arrangement one loses roughly one half of the photoelectrons emitted from the functional layers since they are absorbed by the thick metallic capping stripes. However, the
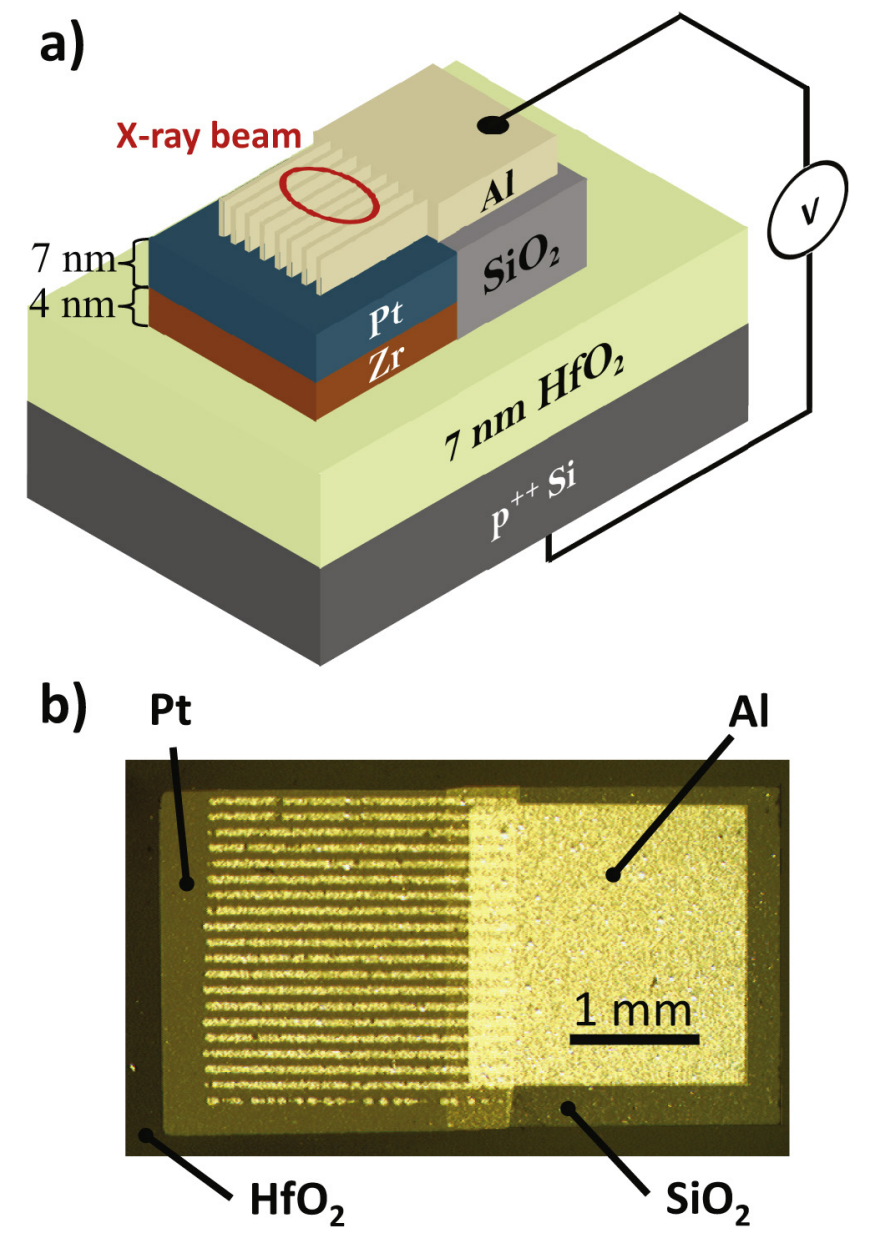

Fig. 1. (a) The schematic structure of the sample with the $\mathrm{p}^{++}-\mathrm{Si} / \mathrm{HfO}_{2} / \mathrm{ZrO}_{x} / \mathrm{Pt}$ functional part prepared for in situ biasing during the HAXPES experiment (see text for more details) and (b) image of the sample used in this experiment. brilliance of the X-ray beam provided at the HAXPES instruments easily compensates for this attenuation. In situ biasing with $U= \pm 0-6 \mathrm{~V}$ was applied to the thus prepared $\mathrm{p}^{++}-\mathrm{Si} / \mathrm{HfO}_{2-x} / \mathrm{ZrO}_{x} / \mathrm{Pt}$ sample and $I-V$ curves were acquired using an Agilent $2912 \mathrm{~A}$ source/measure unit. No electrical forming of the sample was done. During the SET process, no compliance current was applied, due to high levels of leakage currents across the large sample area.

The details of the heteroepitaxial $\mathrm{Pt} / \mathrm{BaTiO}_{3}$ bi-layer growth on $\mathrm{MgO}(100)$ by PLD have been reported elsewhere [21]. Briefly, the bi-layered structures were grown in a single vacuum cycle on (001)-oriented single crystal MgO substrates. An epitaxial Pt layer was grown in ultrahigh vacuum at $T=250^{\circ} \mathrm{C}$. Ultrathin $\mathrm{BaTiO}_{3}$ films were grown on the Pt underlayer at $T=475^{\circ} \mathrm{C}$ in oxygen $\left(P_{\mathrm{O}_{2}} \approx 10^{-3} \mathrm{mbar}\right)$ and further annealed at $T=500^{\circ} \mathrm{C}$ under oxygen with $P_{\mathrm{O}_{2}} \approx 10^{-2}$ mbar. The detailed characterization of the structural [21] and ferroelectric [22] properties of these $\mathrm{Pt} / \mathrm{BaTiO}_{3}$ heterostructures is published elsewhere. Here, we emphasize that a $\sim 10 \mathrm{~nm}$ thick as-grown $\mathrm{BaTiO}_{3}$ layer on the Pt underlayer is found spontaneously polarized (ferroelectric) in a single domain state with the polarization vector pointing toward Pt. To investigate the effect of the oxygen vacancies which are present in the initially grown $\mathrm{BaTiO}_{3}$ layer, $\mathrm{MgO}(100) / \mathrm{Pt} / \mathrm{BaTiO}_{3}$ samples were post-annealed under oxygen with pressures in the range $P_{\mathrm{O}_{2}}=$ $10^{-3}$ to $10^{1}$ mbar.

Using a similar PLD approach, heteroepitaxial $\mathrm{BaTiO}_{3}-$ $(20 \mathrm{~nm}) / \mathrm{La}_{0.67} \mathrm{Sr}_{0.33} \mathrm{MnO}_{3}(200 \mathrm{~nm}) /$ bi-layers were grown on a $\mathrm{SrTiO}_{3}(001)$ substrate.

\section{Results and discussion}

\subsection{Resistive switching in $\mathrm{p}^{++}-\mathrm{Si}_{/} \mathrm{HfO}_{2-x} / \mathrm{ZrO}_{x} /$ Pt stacks}

Typical $I-V$ curves acquired from $\mathrm{p}^{++}-\mathrm{Si} / \mathrm{HfO}_{2-x} / \mathrm{ZrO}_{x} / \mathrm{Pt}$ samples are shown in Fig. 2. The sample exhibits the bipolar switching behavior with the counterclockwise hysteresis. The values of $V_{\text {set }}$ and $V_{\text {reset }}$ obtained from these data are $6 \mathrm{~V}$ and $-4 \mathrm{~V}$, respectively. The derived $I_{\mathrm{on}} / I_{\text {off }}$ ratio, around $10 \%$, is quite low when compared with integrated structures. This is probably due to the high level of leakage current (several orders of magnitude) crossing the large sample area. Nevertheless, $V_{\text {set }}$ and $V_{\text {reset }}$ are the same as those obtained for similar structures $5 \times 10^{-4} \mathrm{~cm}^{2}$ in area $\left(I_{\text {on }} / I_{\text {off }} \sim 100\right)$ indicating that the origin of the switching effect is the same.

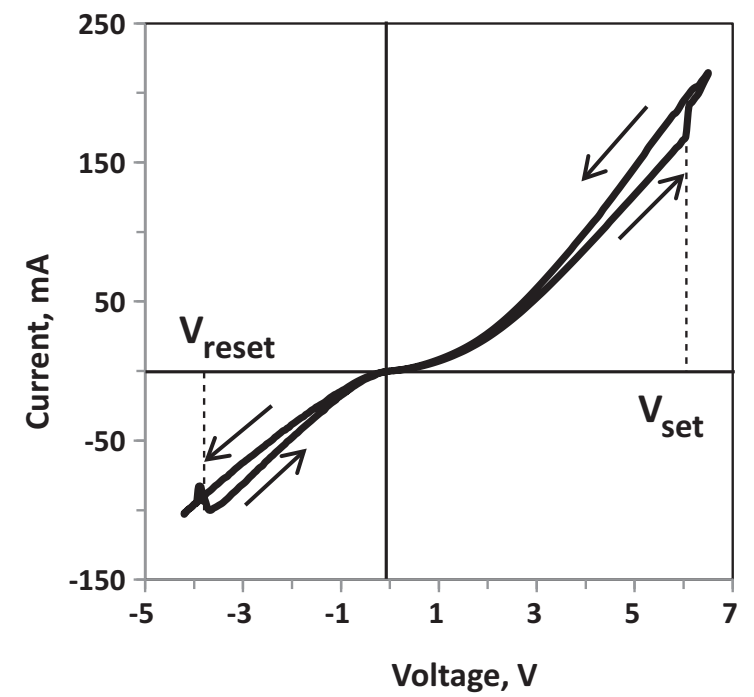

Fig. 2. Typical $I-V$ curve taken from the $\mathrm{p}^{++}-\mathrm{Si} / \mathrm{HfO}_{2} / \mathrm{ZrO}_{x} / \mathrm{Pt}$ sample used for the HAXPES analysis. 
The $\mathrm{Zr} 3 d$ and $\mathrm{Hf} 4 f$ core-level spectra taken from the as-grown $\mathrm{p}^{++}-\mathrm{Si} / \mathrm{HfO}_{2} / \mathrm{Zr} / \mathrm{Pt}$ functional structure are shown in Fig. 3. From the HAXPES spectra it can be concluded that the $4 \mathrm{~nm}$ thick $\mathrm{Zr}$ layer is partially oxidized while $\mathrm{HfO}_{2}$ is reduced. By fitting the line shape of the core level spectra, an oxide-to-metal component intensity ratio $I_{\text {oxide }} / I_{\text {metal }}=95 \%$ for $\mathrm{Hf} 4 \mathrm{f}$ and $32 \%$ for $\mathrm{Zr} 3 d$ is obtained. To verify the natural assumption that the reaction occurs at the $\mathrm{HfO}_{2} / \mathrm{Zr}$ interface, also the deeper lying core levels $\mathrm{Zr} 2 p_{3 / 2}\left(E_{\mathrm{B} Z \mathrm{Zr} 2 p} \approx 2220 \mathrm{eV}\right)$ and $\mathrm{Hf} 3 d\left(E_{\mathrm{B} H \mathrm{Hf} 3 d} \approx 1660 \mathrm{eV}\right)$ were measured (spectra not shown). Due to the smaller kinetic energy and the correspondingly shorter inelastic mean free paths $\lambda$ of the photoelectrons emitted from $\mathrm{Zr}$ $2 p_{3 / 2}$ and $\mathrm{Hf} 3 d_{5 / 2}$ core levels, the spectra are more "surface sensitive" compared to $\mathrm{Zr} 3 d$ and $\mathrm{Hf} 4 f$, respectively [23]. For the "surface sensitive" case, the curve fitting yields $I_{\text {oxide }} / I_{\text {metal }}=96 \%$ for $\mathrm{Hf} 3 d_{5 / 2}$ and $22 \%$ for $\mathrm{Zr} 2 p_{3 / 2}$. By comparing $I_{\text {oxide }} / I_{\text {metal }}$ ratios for the "bulk" versus "surface" sensitive case it is evident that $\mathrm{ZrO}_{x}$ forms at the bottom of the metallic $\mathrm{Zr}$ layer, while $\mathrm{HfO}_{x}$ forms at the top of $\mathrm{HfO}_{2}$ (the information is summarized in Table 1). We conclude that $\mathrm{Zr}$ and $\mathrm{HfO}_{2}$ react at the interface, presumably during deposition of $\mathrm{Zr}$ on top of $\mathrm{HfO}_{2}$.
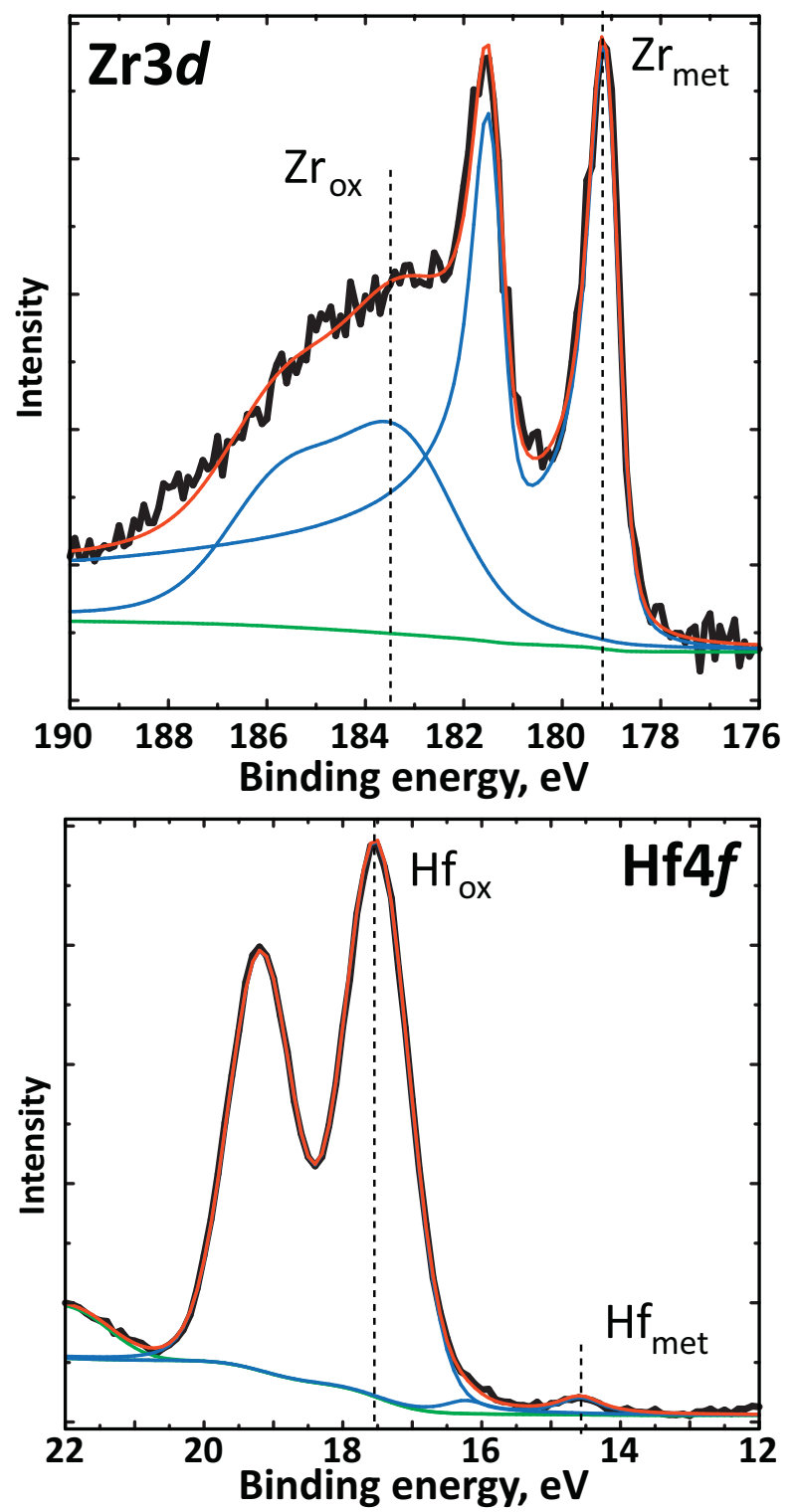

Fig. 3. Core-level $\mathrm{Zr} 3 d$ and $\mathrm{Hf} 4 f$ spectra taken from the as-grown $\mathrm{p}^{++}$ $\mathrm{Si} / \mathrm{HfO}_{2-x} / \mathrm{ZrO}_{x} / \mathrm{Pt}$ functional structure. The X-ray energy was set to $6.0 \mathrm{keV}$.
Table 1

$I_{\text {oxide }} / I_{\text {metal }}$ ratios for the "bulk" (Hf4f and Zr3d) and "surface" (Hf3d and Zr2p) sensitive core level lines.

\begin{tabular}{lcccr}
\hline & Hf3 $d_{5 / 2}$ & Hf4f & Zr2p $p_{3 / 2}$ & Zr3d \\
\hline$E_{\mathrm{B}}, \mathrm{eV}$ & 1660 & 18 & 2200 & 180 \\
$\lambda, \mathrm{nm}[22]$ & $\approx 6.5$ & $\approx 11$ & $\approx 5$ & $\approx 11$ \\
"Off” state, \% & 96 & 95 & 22 & 32 \\
"On” state, \% & 95 & - & 25 & -
\end{tabular}

To investigate the mechanism of the resistive switching effect in the $\mathrm{p}^{++}-\mathrm{Si} / \mathrm{HfO}_{2-x} / \mathrm{ZrO}_{x} / \mathrm{Pt}$ structure, we employ in situ biasing of the sample directly in the HAXPES UHV-chamber. According to previous ex situ current-voltage $(I-V)$ measurements, it is known that the as-grown $\mathrm{p}^{++}-\mathrm{Si} / \mathrm{HfO}_{2-x} / \mathrm{ZrO}_{x} / \mathrm{Pt}$ structure is in a low conductive state (OFF). The in situ pulsed voltage sweep $U=0-6 \mathrm{~V}$, $t_{\text {pulse }}=1 \mu \mathrm{s}$ (where positive voltage is applied to the Pt electrode) results in the resistivity switched to the highly conductive (ON) state as confirmed by $I-V$ measurements (not shown). The measured spectral changes in the $\mathrm{Zr} 2 p_{3 / 2}$ and $\mathrm{Hf} 4 f$ lines for OFF and ON states are shown in Fig. 4. The spectra were fitted by using the model
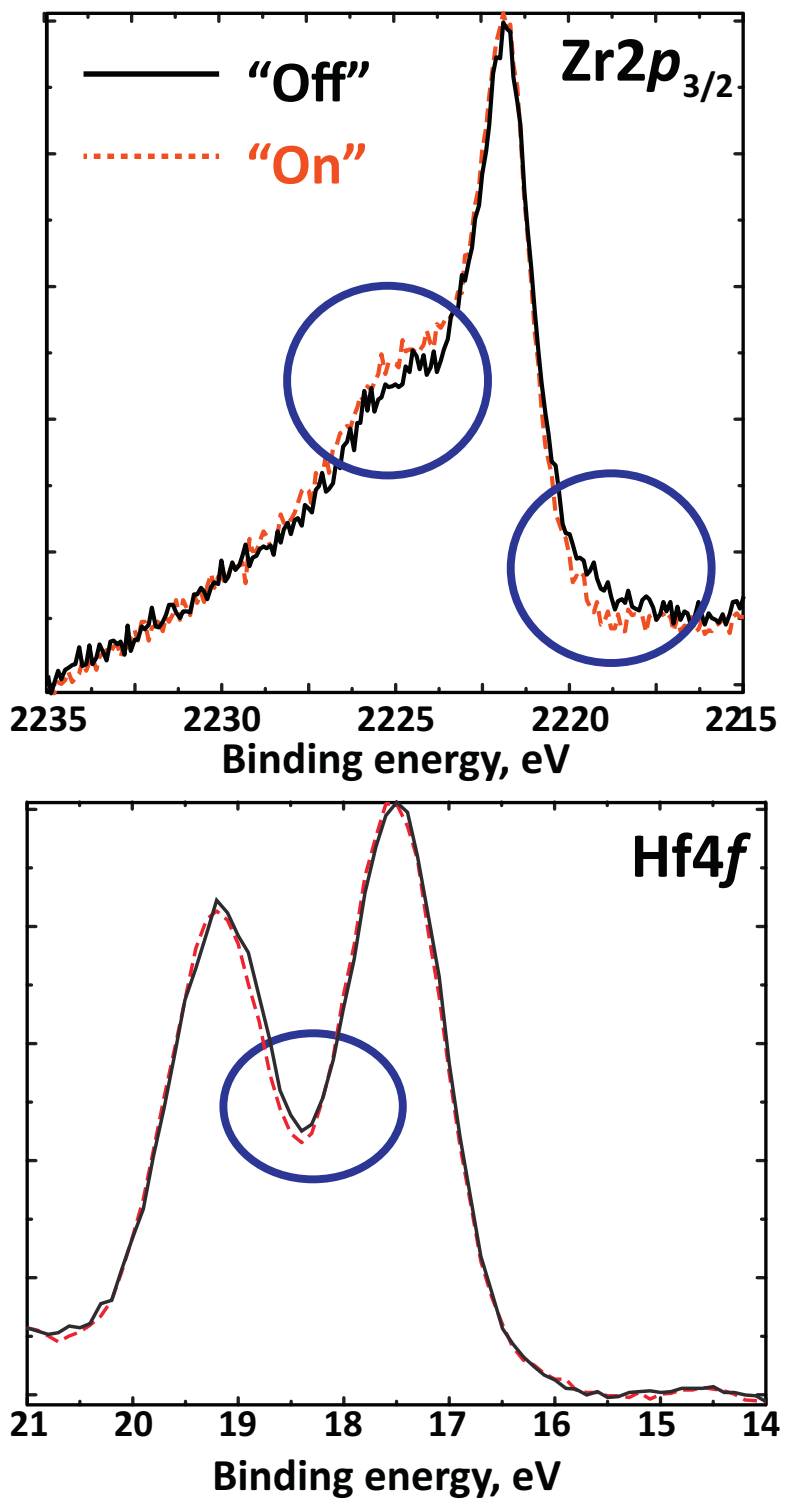

Fig. 4. HAXPES spectra showing the subtle changes in the $\mathrm{Zr} 2 p_{3 / 2}$ and $\mathrm{Hf} 4 f$ core level lines upon resistive switching following in situ biasing of the $\mathrm{p}^{++}-\mathrm{Si} / \mathrm{HfO}_{2-x} / \mathrm{ZrO}_{x} / \mathrm{Pt}$ structure. 


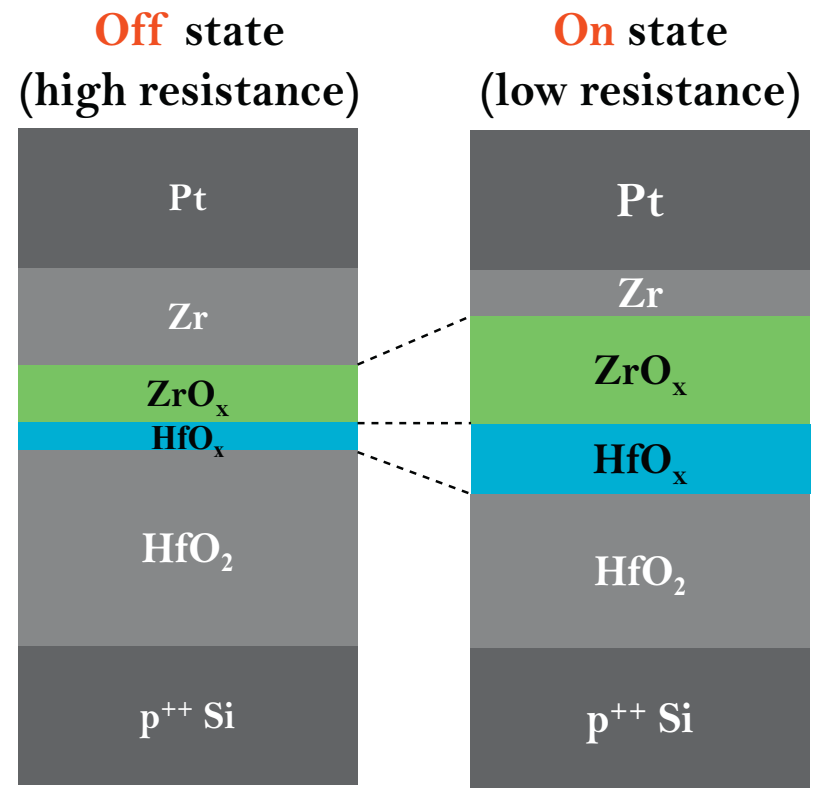

Fig. 5. Schematic of the chemical evolution of the $\mathrm{p}^{++}-\mathrm{Si} / \mathrm{HfO}_{2-x} / \mathrm{ZrO}_{x} / \mathrm{Pt}$ stack upon resistive switching as obtained from the HAXPES analysis following in situ biasing.

ascribing their evolution to the changes of the $I_{\text {oxide }} / I_{\text {metal }}$ ratio for both lines.

In particular, for the $\mathrm{Zr} 2 p_{3 / 2}$ line $I_{\text {oxide }} / I_{\text {metal }}$ increases from $22 \%$ to $25 \%$, while for Hf $4 f$ the $I_{\text {oxide }} / I_{\text {metal }}$ ratio decreases slightly from $96 \%$ to $95 \%$. Although these changes are rather small they are indicating that the switching from OFF to ON corresponds to a thickness reduction of $\mathrm{HfO}_{x}$ and simultaneous increasing of the $\mathrm{ZrO}_{x}$ layers at the interface. This evolution of the interface chemistry is schematically illustrated in Fig. 5. According to this model, the $\mathrm{HfO}_{2}$ layer adjacent to the interface with $\mathrm{Zr}$ is rich in oxygen vacancies due to the initial partial oxidation (reduction) of $\mathrm{Zr}\left(\mathrm{HfO}_{2}\right)$ to form $\mathrm{ZrO}_{x}\left(\mathrm{HfO}_{2-x}\right)$. During in situ positive biasing the positively charged oxygen vacancies are driven away from $\mathrm{HfO}_{2}$ at the interface effectively enriching it with oxygen and thus facilitating further oxidation of $\mathrm{Zr}$. The latter process produces more oxygen vacancies in $\mathrm{HfO}_{2}$ and dramatically increases the conductivity (ON state). The biasing of the opposite sign results in the reversed sequence of reduction/oxidation steps as confirmed by HAXPES (spectra not shown).

The observed effects at the $\mathrm{HfO}_{2-x} / \mathrm{ZrO}_{x}$ interface support the model of an oxidation/reduction mechanism of the resistive switching in these bilayers. However, the fact that the changes particularly in the $\mathrm{HfO}_{2}$ layer are quite small implies that the switching occurs due to a localized rather than extended area phenomenon. We believe that the generation of the (charged) oxygen vacancies at the $\mathrm{HfO}_{2-x} / \mathrm{ZrO}_{x}$ interface and their further drift following the positive (negative) biasing of the stack results in the formation ("dissolution") of the localized conductive paths (filaments) which define the resistance switching across the stack.

\subsection{Conduction band offset in $\mathrm{Pt} / \mathrm{BaTiO}_{3}$}

Following the HAXPES methodology described above, we determined the valence band offset at the $\mathrm{Pt} / \mathrm{BaTiO}_{3}$ heteroepitaxial interface by measuring the line separations $\quad\left(E_{\mathrm{Pt} 4 f 7 / 2}-E_{\mathrm{F}}\right)_{\mathrm{Pt}}=71.04 \pm 0.05 \mathrm{eV}, \quad\left(E_{\mathrm{Ti} 2 p 3 / 2}-\mathrm{VBM}\right)_{\mathrm{BTO}}=$ $455.71 \pm 0.05 \mathrm{eV}$ and $\left(E_{\mathrm{Pt} 4 f 7 / 2}-E_{\mathrm{Ti} 2 p 3 / 2}\right)_{\mathrm{BTO} / \mathrm{Pt}}=387.61 \pm 0.05 \mathrm{eV}$. Using formula (1), we then get $\mathrm{VBO}_{\mathrm{Pt} / \mathrm{BTO}}=2.94 \pm 0.05 \mathrm{eV}$ (more detailed information is published elsewhere [22]). To determine the electric potential step height $\varphi_{\mathrm{Pt} / \mathrm{BTO}}$ (also known as conduction

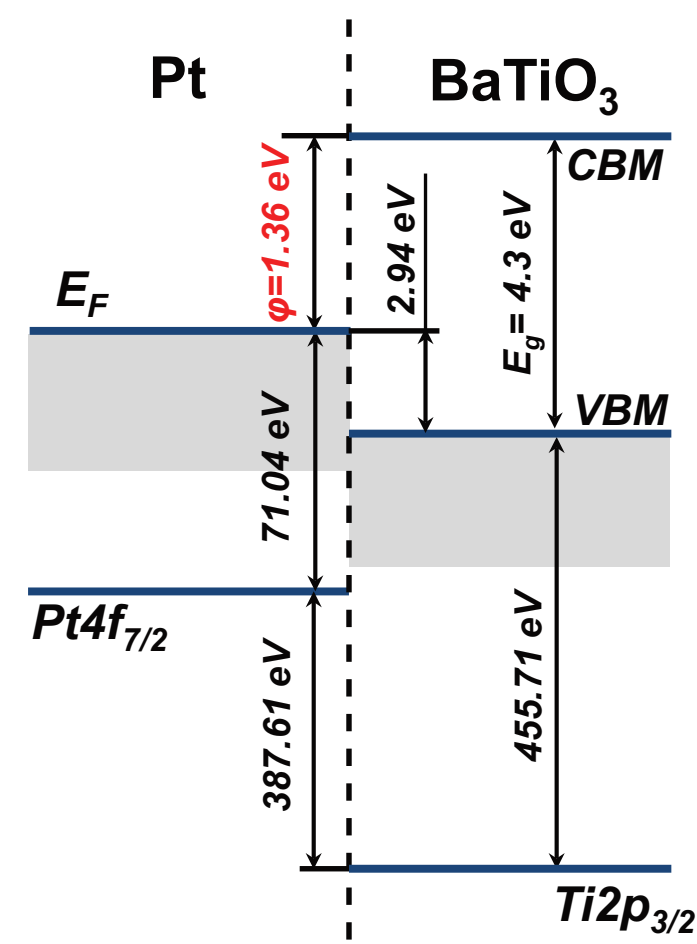

Fig. 6. Electronic band diagram at the $\mathrm{Pt} / \mathrm{BaTiO}_{3}$ interface as determined by in situ HAXPES in combination with ex situ REELS techniques.

band offset, $\mathrm{CBO}$ ), the band gap $E_{\mathrm{g}}$ for the investigated ultrathin heteroepitaxial $\mathrm{BaTiO}_{3}$ films were determined by ex situ reflection electron energy loss spectroscopy (REELS) [21] to be $E_{\mathrm{g}}=4.3 \mathrm{eV}$. Therefore, the conduction band offset at the $\mathrm{Pt} / \mathrm{BaTiO}_{3}$ interface is $\varphi_{\mathrm{Pt} / \mathrm{BTO}}=1.36 \pm 0.05 \mathrm{eV}$. The band line-up obtained using these values is shown in Fig. 6. We note that according to recent data on the ferroelectric properties of heteroepitaxial $\mathrm{BaTiO}_{3}$ layers grown on a Pt underlayer, they are spontaneously polarized downward [22], and therefore the potential step is measured for the $\mathrm{BaTiO}_{3}$ layer polarization oriented toward Pt. Since the $\mathrm{BaTiO}_{3}$ layer is polarized, one would expect a potential distribution across the film thickness and subsequently a broadening of the spectral lines taken from the constituents of the ferroelectric layer. However, the HAXPES core level data show no line broadening in the functional ferroelectrics. According to experimental work [24], surface adsorbates play a governing role in the potential formation mechanism and completely screen the polarization charge on the open surface of the ferroelectric. Therefore, polarization in the structurally perfect ferroelectric results in a HAXPES line shift due to the formation of electric dipoles at the interface (unless adsorbates are removed by in situ mild heating in UHV-the effect will be reported elsewhere).

Another effect which can alter both the line width of HAXPES spectra taken from a ferroelectric and the electronic conditions at the metal/ferroelectric interface is the presence of fixed and/or mobile charges in the ferroelectric. These charges are particularly associated with oxygen vacancies which are the prevailing defects in complex metal oxide films once they are put in contact with metal electrodes [25]. To investigate the possible effect of oxygen vacancies present in $\mathrm{BaTiO}_{3}$ on the band line-up in contact with $\mathrm{Pt}$, heteroepitaxial $\mathrm{BaTiO}_{3}$ layers were grown in vacuum $\left(P_{\mathrm{O}_{2}}=10^{-7} \mathrm{mbar}\right)$ and further post-annealed under oxygen with different pressures. The changes of the measured $\mathrm{Pt} / \mathrm{BaTiO}_{3}$ valence band offset and the Ba $4 d$ and Ti $2 p$ core level line width as a function of $\mathrm{O}_{2}$ pressure during annealing are shown in Fig. 7a and b, respectively. 

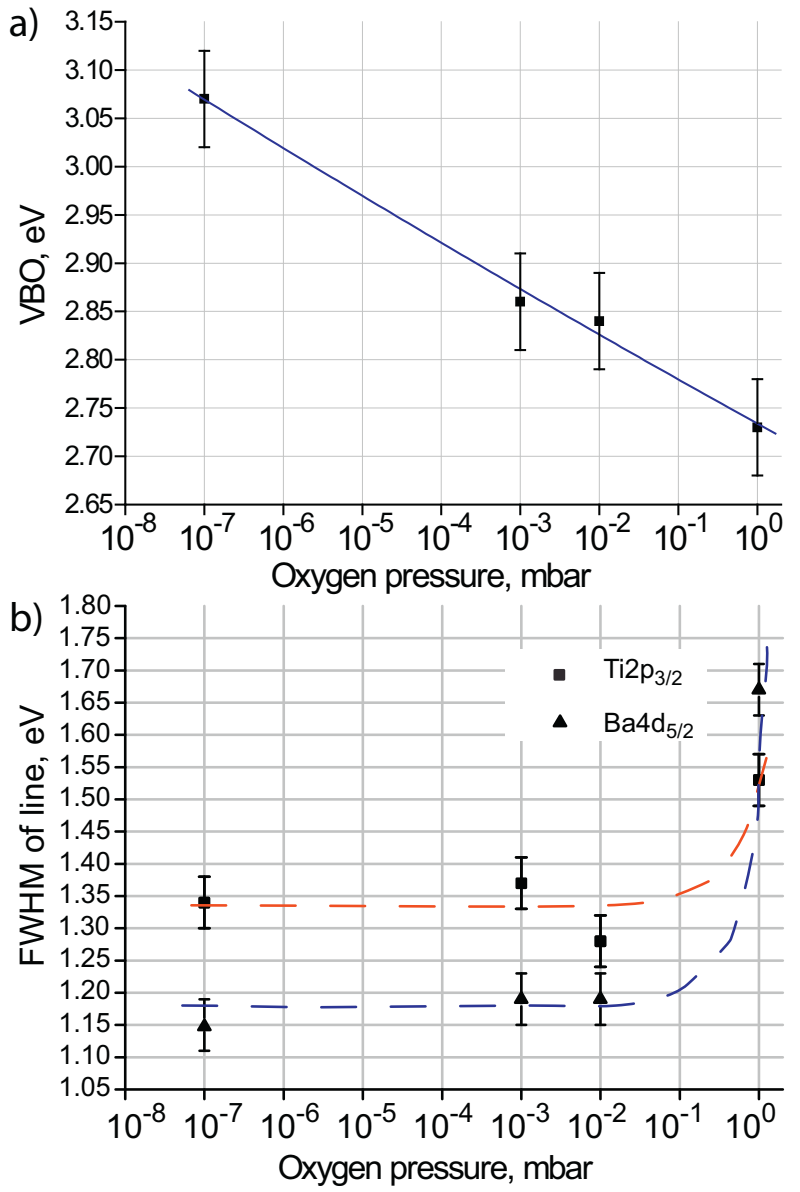

Fig. 7. $\mathrm{BaTiO}_{3}$ valence band offset with respect to $\mathrm{Pt}(\mathrm{a})$ and half-width of $\mathrm{Ti} 2 \mathrm{p}_{3 / 2}$ and $\mathrm{Ba}_{4} \mathrm{~d}_{5 / 2}$ lines (b) as a function of $\mathrm{O}_{2}$ pressure during the post-annealing of the $\mathrm{MgO} / \mathrm{Pt} / \mathrm{BaTiO}_{3}$ heterostructures $\left(T=500^{\circ} \mathrm{C}, t=30 \mathrm{~min}\right)$ as measured by HAXPES

The derived evolution of the electronic structure of the $\mathrm{Pt} / \mathrm{BaTiO}_{3}$ interface corroborates the model describing the formation of the electric dipole at the metal/dielectric interface in terms of electron transfer across the interface from oxygen vacancies $\left(V_{O}\right)$ in the ferroelectric - acting as donors - to the metal, once the metal work function is sufficiently large [26]. Assuming that the energy levels of (both neutral and charged) oxygen vacancies in $\mathrm{BaTiO}_{3}$ lie in the upper part of the band gap, the high $V_{O}$ concentration in as-grown $\mathrm{BaTiO}_{3}$ results in a decrease of the "effective" work function of Pt in contact giving rise to the larger VBO. The concentration of $V_{\mathrm{O}}$ in $\mathrm{BaTiO}_{3}$ presumably decreases monotonously depending on the oxygen pressure during the post-annealing, and the VBO subsequently increases.

Up to $0.3 \mathrm{eV}$ change was predicted in the $\mathrm{Pt} / \mathrm{SrTiO}_{3} \mathrm{Schottky}$ barrier height depending on the number of $V_{\mathrm{O}}$ [25], while similar calculations reveal up to $1 \mathrm{eV}$ for the Pt/PZT system as a function of $V_{\mathrm{O}}$ concentration [27]. In this experiment, the observed change of $\mathrm{VBO}_{\mathrm{Pt} / \mathrm{BTO}}$ of $\sim 0.3 \mathrm{eV}$ depending on the oxygen pressure during annealing is well within this range. Previously, a similar effect on the band line-up at the $\mathrm{Fe} / \mathrm{BaTiO}_{3}$ interface was observed depending on the top $\mathrm{BaTiO}_{3}$ layer growth conditions [18].

The observed $\mathrm{Ba} 4 d$ and $\mathrm{Ti} 2 p$ core level line broadening in $\mathrm{BaTiO}_{3}$ following the increase of the oxygen pressure during the annealing is explained by the removal of the mobile (charged) oxygen vacancies which contribute to the screening of the electric field inside the ferroelectric layer.

\subsection{Conduction band offset in $\mathrm{LSMO} / \mathrm{BaTiO}_{3}$}

The analogous HAXPES methodology was adopted to reconstruct the band line-up at the $\mathrm{LSMO} / \mathrm{BaTiO}_{3}$ interface, where the conductive LSMO layer is treated as a metal. The line separations $\left(E_{\mathrm{Sr} 2 s}-E_{\mathrm{F}}\right)_{\mathrm{LSMO}}=2215.67 \mathrm{eV}, \quad\left(E_{\mathrm{Ti} 2 p 3 / 2}-V B M\right)_{\mathrm{BTO}}=455.71 \pm 0.05 \mathrm{eV}$ and $\left(E_{\mathrm{Sr} 2 s}-E_{\mathrm{Ti} 2 p 3 / 2}\right)_{\mathrm{LSMO} / \mathrm{BTO}}=1756.67 \mathrm{eV}$ were measured for the single LSMO layer and $\mathrm{BaTiO}_{3}$ layers on a $\mathrm{SrTiO}_{3}$ substrate, and

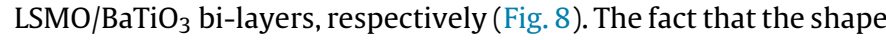
of $\mathrm{Ti} 2 \mathrm{p}$ line taken from the $\mathrm{BaTiO}_{3}$ layer exactly coincides with that from the $\mathrm{BaTiO}_{3}$ reference sample evidences the absence of space charge in the bulk of $\mathrm{BaTiO}_{3}$ layer. Using formula (1) adapted to the $\mathrm{LSMO} / \mathrm{BaTiO}_{3}$ system, we calculate the valence band offset $\mathrm{VBO}_{\mathrm{LSMO} / \mathrm{BTO}}=3.29 \pm 0.05 \mathrm{eV}$, and assuming again the band gap in ultrathin BTO layers $E_{\mathrm{g}}=4.3 \mathrm{eV}$, we get $\mathrm{CBO}_{\mathrm{LSMO} / \mathrm{BTO}}=1.01 \pm 0.05 \mathrm{eV}$ (Fig. 9). The obtained value of the conduction band offset at the $\mathrm{LSMO} / \mathrm{BaTiO}_{3}$ interface is considerably smaller compared to that at the $\mathrm{Pt} / \mathrm{BaTiO}_{3}$ interface $\left(\mathrm{CBO}_{\mathrm{Pt} / \mathrm{BaTiO}_{3}}=1.36 \mathrm{eV}\right)$. These results are consistent considering much larger vacuum work function values of the noble metals such as $\mathrm{Pt}\left(\mathrm{WF}_{\mathrm{Pt}}=5.1 \mathrm{eV}\right)$ as compared to that of the (conducting) oxides which may be as low as $\sim 4 \mathrm{eV}$. Even taking into account the "pinning" effect described above which often occurs at the metal oxide/Pt interface, the effective work function of $\mathrm{Pt}$ in contact with $\mathrm{BaTiO}_{3}$ appears to be larger than that of LSMO.

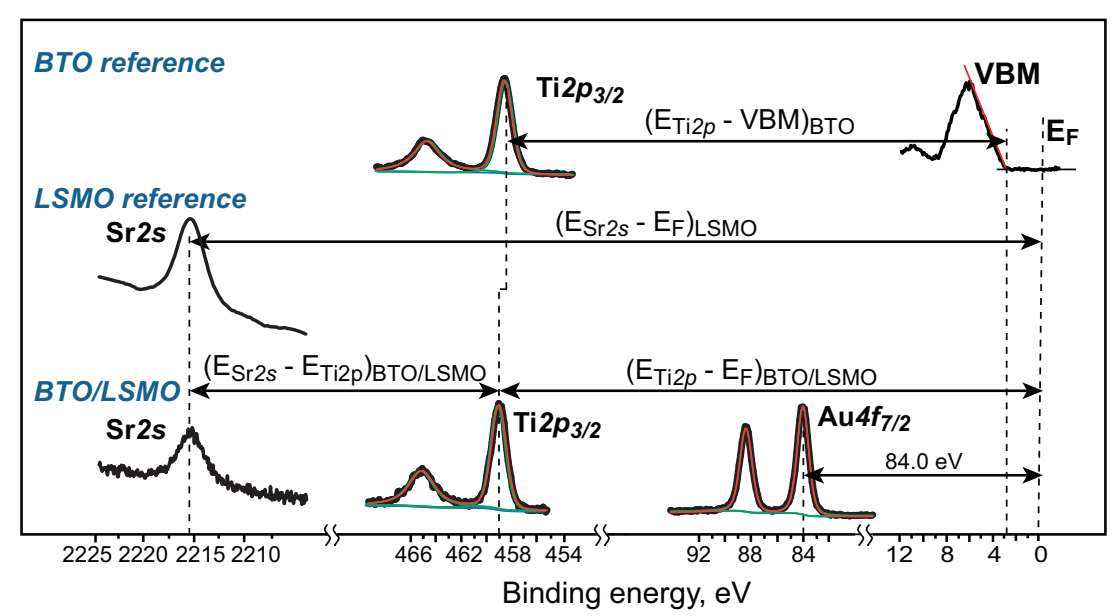

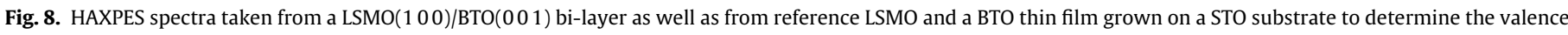
band offset in the LSMO/BTO heterostructure. 


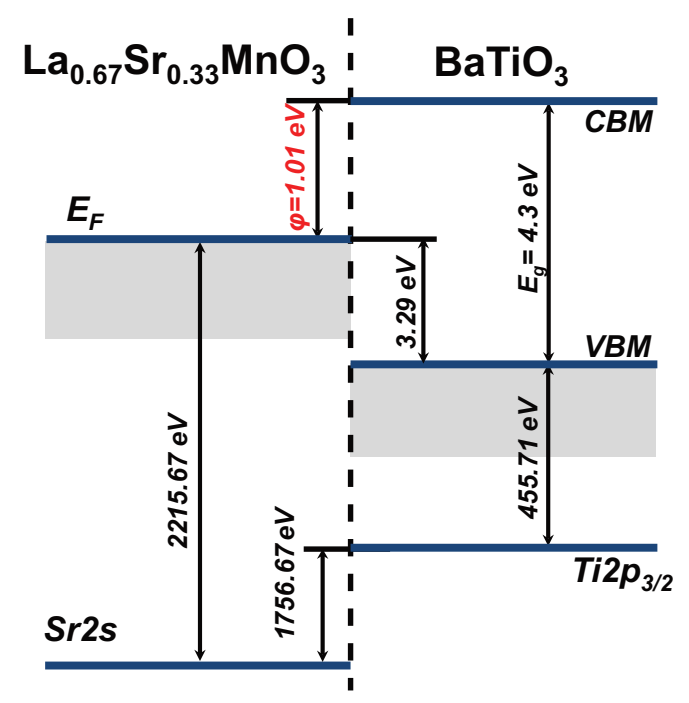

Fig. 9. Electronic band diagram at the $\mathrm{LaSr}_{0.67} \mathrm{Sr}_{0.33} \mathrm{O}_{3} / \mathrm{BaTiO}_{3}$ interface as determined by in situ HAXPES in combination with ex situ REELS techniques.

\section{Conclusion}

The HAXPES technique has been successfully employed to characterize the chemical and electronic properties of buried interfaces in functional multilayered structures. Using the developed methodology, we were able to monitor in situ the changes in both the chemical and electronic structure at the metal/insulator interface of $\mathrm{p}^{++}-\mathrm{Si} / \mathrm{HfO}_{2-x} / \mathrm{ZrO}_{x} / \mathrm{Pt}$ stacks accompanying the reversible resistive switching effect. The electronic band alignment and particularly the electric potential barrier height were experimentally determined at the $\mathrm{Pt} / \mathrm{BaTiO}_{3}$ and $\mathrm{LSMO} / \mathrm{BaTiO}_{3}$ interfaces. An additional result obtained for $\mathrm{Pt} / \mathrm{BaTiO}_{3}$ is that oxygen vacancies present in the ultrathin $\mathrm{BaTiO}_{3}$ layer affect the interfacial electronic properties. It is still demanding to directly correlate the spectral information provided by HAXPES with the electronic and electrical (transport) properties of the functional structures modeling the operation of the real devices. The particular challenge, however, is to quantify changes of the electronic conditions at the metal/FE interfaces upon polarization reversal in ferroelectric tunnel junctions, or to monitor the evolution of the chemical and/or electronic structure during the resistive switching in metal-insulator-metal trilayers.

\section{Acknowledgements}

The expert support of the staff at DORIS III beamline BW2 and PETRA III beamline P09 is gratefully acknowledged. The HAXPES instrument at beamline P09 is jointly operated by the University of Würzburg (R. Claessen), the University of Mainz (C. Felser) and DESY. We would like to thank Ksenia Maksimova (E. Kant Baltic Federal University, Russia) for the assistance in the growth of $\mathrm{Pt} / \mathrm{BaTiO}_{3} / \mathrm{MgO}\left(\begin{array}{lll}0 & 0 & 1\end{array}\right)$ and $\mathrm{BTO} / \mathrm{LSMO} / \mathrm{STO}\left(\begin{array}{lll}0 & 0 & 1\end{array}\right)$ heteroepitaxial samples. This work was financially supported by the Russian Ministry of Education and Science (Contract No. 11.519.11.3007) and the German Federal Ministry of Education and Research(BMBF) under contracts 05KS7UM1, 05K10UMA, 05KS7WW3, 05K10WW1 and $05 \mathrm{~K} 10 \mathrm{CHB}$.

\section{References}

[1] Special issue on "Materials for Electronics", Science 327 (2010).

[2] K. Kobayashi, NIMA 601 (2009), 32.

[3] T. Nagata, M. Haemori, Y. Yamashita, H. Yoshikawa, Y. Iwashita, K. Kobayashi, T. Chikyow, APL 97 (2010), 082903.

[4] T. Nagata, M. Haemori, Y. Yamashita, H. Yoshikawa, Y. Iwashita, K. Kobayashi, T. Chikyow, APL 99 (2011), 223517.

[5] R. Claessen, M. Sing, M. Paul, G. Berner, A. Wetscherek, A. Müller, W. Drube, New J. Phys. 11 (2009), 125007.

[6] R. Waiser, R. Dittmann, G. Staikov, K. Szot, Adv. Mater. 21 (2009) 2632.

[7] J. Lee, E.M. Bourim, W. Lee, J. Park, M. Jo, S. Jung, J. Shin, H. Hwang, APL 97 (2010) 172105.

[8] P. Calka, E. Martinez, D. Lafond, S. Minoret, S. Tirano, B. Detlefs, J. Roy, J. Zegenhagen, C. Guedj, J. Appl. Phys. 109 (2011) 124506.

[9] M.Ye. Zhuravlev, R.F. Sabirianov, S.S. Jaswal, E.Y. Tsymbal, Phys. Rev. Lett. 94 (2005) 246802

[10] A. Gruverman, D. Wu, H. Lu, Y. Wang, H.W. Jang, C.M. Folkman, M.Ye. Zhuravlev, D. Felker, M. Rzchowski, C.-B. Eom, E.Y. Tsymbal, Nano Lett. 9 (2009) 3539.

[11] A. Chanthbouala, A. Crassous, V. Garcia, K. Bouzehouane, S. Fusil, X. Moya, J. Allibe, B. Dlubak, J. Grollier, S. Xavier, et al., Nat. Nanotechnol. 7 (2012) 101.

[12] K.J. Choi, M. Biegalski, Y.L. Li, A. Sharan, J. Schubert, R. Uecker, P. Reiche, Y.B. Chen, X.Q. Pan, V. Gopalan, L.-Q. Chen, D.G. Schlom, C.B. Eom, Science 306 (2004) 1005.

[13] C.-G. Duan, S.S. Jaswal, E.Y. Tsymbal, Phys. Rev. Lett. 97 (2006) 047201.

[14] V. Garcia, M. Bibes, L. Bocher, S. Valencia, F. Kronast, A. Crassous, X. Moya, S. Enouz-Vedrenne, A. Gloter, D. Imhoff, et al., Science 327 (2010) 1106.

[15] A. Gloskovskii, G. Stryganyuk, G.H. Fecher, C. Felser, S. Thiess, H. Schulz-Ritter, W. Drube, G. Berner, M. Sing, R. Claessen, M. Yamamoto, J. El. Spec. Rel. Phenom. 185 (2012) 47.

[16] http://unifit-software.de.

[17] E.A. Kraut, R.W. Grant, J.R. Waldrop, S.P. Kowalczyk, Phys. Rev. Lett. 44 (1980) 1620.

[18] A. Zenkevich, R. Mantovan, M. Fanciulli, M. Minnekaev, Yu.A. Matveyev, Yu.Yu. Lebedinskii, S. Thiess, W. Drube, Appl. Phys. Lett. 99 (2011) 182905.

[19] Yu. Matveyev, A. Zenkevich, Yu. Lebedinskii, S. Thiess, W. Drube, Microelectr. Eng. 88 (2011) 1353.

[20] A. Zenkevitch, I. Khabelashvili, J. Chevallier, Thin Solid Films 311 (1997) 119.

[21] A. Zenkevich, M. Minnekaev, Yu. Lebedinskii, K. Bulakh, A. Chouprik, A. Baturin, R. Mantovan, M. Fanciulli, O. Uvarov, Thin Solid Films 520 (2012) 4586.

[22] A. Zenkevich, Minnekaev M., Yu. A. Matveyev, Yu. Lebedinskii, K. Bulakh, A. Chouprik, K. Maksimova, A. Baturin, S. Thiess, W. Drube, Appl. Phys. Lett. 102 (2013) 062907

[23] In this work we adopt $\lambda$ values for the specified core level photoelectrons in $\mathrm{HfO}_{2}\left(\mathrm{ZrO}_{2}\right)$ the same as those previously measured for $\mathrm{SiO}_{2}$ layer for similar kinetic energies [18] (see Table 1), and the sampling depths are thus estimated $\sim 10 \mathrm{~nm}$ and $\sim 22 \mathrm{~nm}$ for the "bulk" and "surface sensitive" cases, respectively.

[24] S.V. Kalinin, D.A. Bonnell, Phys. Rev. B 63 (2001) 125411.

[25] J. Robertson, O. Sharia, A. Demkov, App. Phys. Lett. 91 (2007) 132912.

[26] M. Dawber, K.M. Rabe, J.F. Scott, Rev. Mod. Phys. 77 (2005) 1083

[27] M. Dawber, J.F. Scott, Int. Ferroelectr. 38 (2001), 161. 